\title{
Experimental Investigation of a Synthetic Jet Array in a Laminar Channel Flow
}

\author{
P. Dančová ${ }^{1,2}, Z$. Trávníček ${ }^{2}$, and T. Vít ${ }^{1,2}$ \\ ${ }^{1}$ Technical University of Liberec, FS, KEZ, Studentská 2, 46117 Liberec 1, Czech Republic \\ ${ }^{2}$ Instutite of Thermomechanics ASCR, v.v.i., Dolejškova 1402/5, 18200 Praha 8, Czech Republic
}

\begin{abstract}
The paper deals with an impinging synthetic jet, namely on the case of a synthetic jet array interacting with a laminar channel flow. This arrangement can be useful in many micro-scale applications, such as cooling of micro-electronics. The flow regime in micro-scale is usually laminar with very small Reynolds numbers; therefore synthetic jet array can be used for the profile disturbance and heat transfer enhancement. The paper focuses on the low Reynolds number (in order $10^{2}$ ). The working fluid is water and a piezoceramic transducer is used as a moving membrane in the synthetic jet actuator. Experiments are performed with four experimental methods (tin ion visualization, hot wire anemometry in constant temperature mode, laser Doppler vibrometry and particle image velocimetry) in three laboratories (at the Eindhoven University of Technology, Netherlands, at the Institute of Thermodynamics CAS, v.v.i. and Technical University of Liberec, both Czech Republic).
\end{abstract}

\section{Introduction}

The motivation behind this work is to demonstrate an arrangement in which a synthetic jet array interacting with a laminar channel flow can be useful for micro-scale applications, such as cooling in micro-electronics. The flow regime in micro-scale is usually laminar with very small Reynolds numbers and cooling is typically based on gradient diffusion. Therefore, this study focuses on low Reynolds numbers; more specifically, current Reynolds numbers are so low that they cannot initiate a transition to turbulence. Therefore, a synthetic jet array is used as a disturbance of laminar channel flow. A potential application of this method is to increase heat transfer using the flow control of a main flow.

\section{Component tasks}

\subsection{Channel flow}

As was mentioned above, this work focuses on low Reynolds numbers. In other words, on the laminar channel flow. The flow is supposed as two-dimensional case as a flow between two parallel walls. Because of a $2 \mathrm{D}$ simplification, the ration of mean and maximal velocity is (see [1])

$$
\frac{v_{\mathrm{M}}}{v_{\max }}=\frac{2}{3} \text {. }
$$

\subsection{Synthetic jet}

A synthetic jet (SJ) is generated by the periodic motion of an actuator oscillating membrane. A SJ is synthesized by the interactions within a train of vortex rings or counterrotating vortex pairs in axis-symmetric or twodimensional geometry; see Smith and Glezer [2]. Vortex rings are formed at the lip of the orifice (see figure 1). These rings move in $y$ direction with a velocity, which must be high enough to prevent interaction with suction in the orifice. It was observed that an SJ far enough from the orifice has a character of a conventional steady jet. This is caused by the development and dissipation of vortexes. One of the main advantages of a SJ is that the time-mean mass flux of the oscillatory flow in the orifice is zero; hence the other common expression is a zero-netmass-flux (ZNMF) jet. A ZNMF eliminates the requirement of a blower and piping for the fluid inlet. Though the SJ actuator works with a ZNMF at the orifice, the momentum of the resultant SJ at a specific distance from the lip in $y$ is non-zero [2-4].

A SJ has many significant applications and the number of applications is increasing all the time. The most important applications can be divided into two main groups:

- Main (primary) flow control (jet vectoring [5, 6], flow field control in external or internal aerodynamics [7], increasing heat transfer due to the main flow control [8, 9]). 


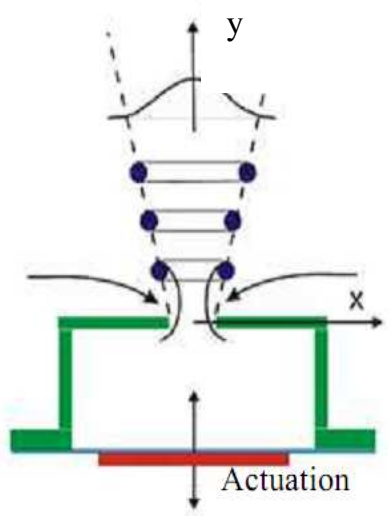

Fig. 1. Basic principle of SJ.

- The use of a stand-alone SJ or system of synthetic jets (SJ used for intensification of heat transfer [10, 11].

\subsection{Problem parameterization}

A SJ is characterized by several independent parameters. When considering the plug flow model (one-dimensional piston - like flow in the actuator orifice), the main parameters of a SJ are:

- The actuator orifice diameter $D$.

- The time-mean orifice velocity $U_{0}$

$$
U_{0}=\frac{1}{T} \int_{0}^{T_{\mathrm{E}}} u_{0}(t) d t,
$$

where $T$ is the time period, i.e. $T=1 / f$ and $f$ is the frequency, $T_{\mathrm{E}}$ is the extrusion time $\left(T_{\mathrm{E}}=T / 2\right.$ for the sinusoidal waveform $), u_{0}(t)$ is the periodical axial orifice velocity. For the sinusoidal waveform $u_{0}(t)=U_{\max } \cdot \sin (2 f t)$, one can easily derive $U_{\max }=. U_{0}$.

- The "stroke length" $L_{0}$

$$
L_{0}=U_{0} T=\frac{U_{0}}{f} .
$$

- The Reynolds number of a SJ

$$
\operatorname{Re}_{\mathrm{SJ}}=\frac{U_{0} D}{}
$$

where $v$ is the kinematic viscosity.

- The Strouhal number is not an independent parameter; it depends above parameters (Eq. (3)). For sinusoidal waveform

$$
S t=\frac{1}{U_{0}}=\frac{1}{L_{0}} .
$$

- Because the investigation is focused on a channel flow influenced by an array of SJs, it is also necessary to define the Reynolds number of the 2D channel flow (2D simplification of the flow between two parallel plates)

$$
\operatorname{Re}_{\mathrm{C}}=\frac{U_{\mathrm{C}} \quad D_{\mathrm{CH}}}{U_{\mathrm{C}} 2 H},
$$

where $U_{\mathrm{C}}=(2 / 3) U_{\mathrm{C} \_ \text {max }}$ is the time-mean velocity through the channel, $U_{\mathrm{C}_{-} \max }$ is the maximum velocity in the channel, and $H$ is the channel height.

- The strength of the control SJs can be quantified relative to the main channel flow in terms of the ratios of velocities, flow rates, and momentum

$$
c_{\mathrm{U}}=\frac{1}{n} \sum_{i=1}^{n}\left(\frac{U_{0 \mathrm{i}}}{U_{\mathrm{C}}}\right),
$$

$$
c_{\mathrm{Q}}=\frac{A}{A_{\mathrm{C}}} \sum_{n=1}^{n}\left(\frac{U_{0 \mathrm{i}}}{U_{\mathrm{C}}}\right) \text {, }
$$

$$
c_{\mathrm{M}}=\frac{5^{2}}{24}\left(\frac{A}{A_{\mathrm{C}}}\right) \sum_{i=1}^{n}\left(\frac{U_{0 \mathrm{i}}}{U_{\mathrm{C}}}\right)^{2},
$$

where $A$ is the cross-section area of the SJ actuator orifice $\left(A=\pi D^{2} / 4\right), n$ is the number of control SJs $(n=4$ for the current study), and $A_{\mathrm{C}}=B H$ is the cross section of the channel (width $\mathrm{x}$ height). In Equation (9), the laminar parabolic profile of two-dimensional flow is assumed.

\section{Experimental setup and methods}

\subsection{Experimental setup}

\subsubsection{Synthetic jet actuator}

The first step of the SJ research was the development of a SJ actuator. The actuator (see figure 2) is made from Plexiglas and consists of a sealed cavity, which was equipped with an emitting orifice (diameter $D=3.0 \mathrm{~mm}$ ) and with an actuating piezoelectric membrane (KINGSTATE KPS-100, diameter of the cavity $\left.D_{\mathrm{D}}=36 \mathrm{~mm}\right)$.

\subsubsection{Synthetic jet array setup}

A SJ array was four SJ actuators placed across the channel cross section. For the investigation, two experimental setups were made. The first setup (see figure 3) was made for LDV experiments to find four PCTs with the same characteristics and phase shift and to carry out flow visualization and PIV experiments of an SJ array without affecting the channel flow. This setup was made from Plexiglas. The upper wall is bolted on with four screws, allowing the wall to be removed.

For the experiments with a SJ array in a channel, a Plexiglas wall (figure 4) with dimensions: length (length with start) $\mathrm{x}$ width $\mathrm{x}$ thickness $=(860(880) \times 200$ $\mathrm{x} 10) \mathrm{mm}$ was constructed. The wall inlet was carefully rounded, and the SJ array was placed $520 \mathrm{~mm}$ from the beginning. The wall was put into a circulation water 

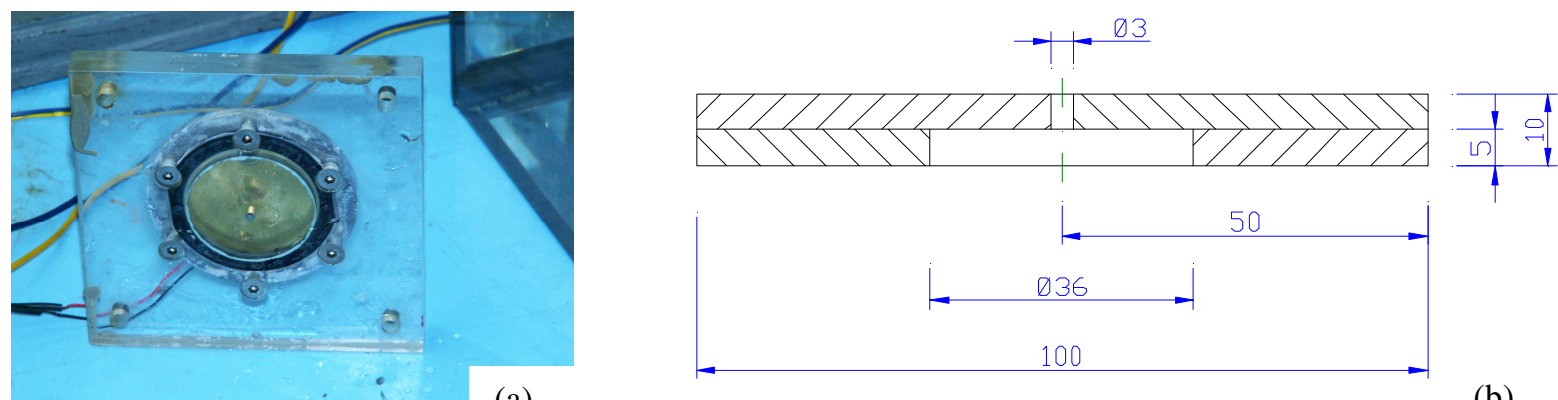

(a)

(b)

Fig. 2 SJ actuator (a) general view, (b) schematic view.

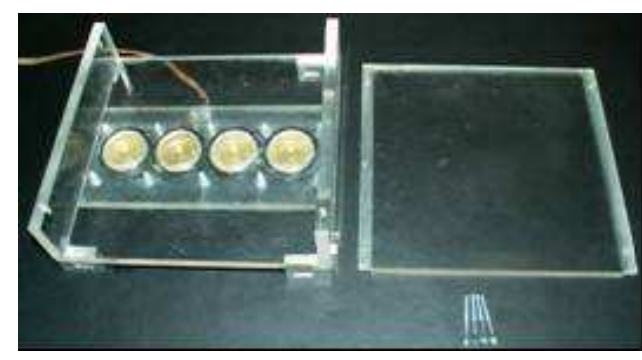

(a)
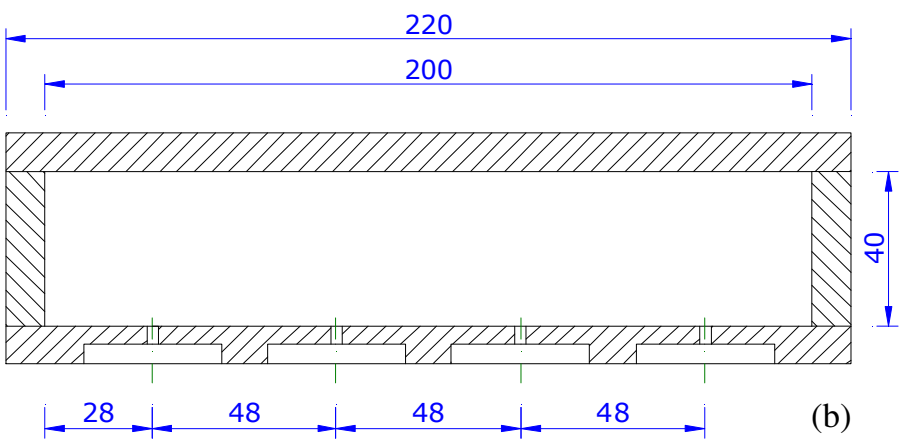

Fig. 3 SJ array device (a) general view, (b) schematic view.

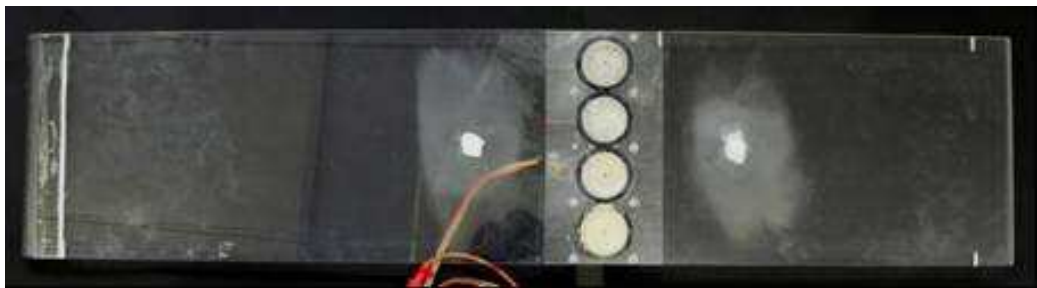

(a)

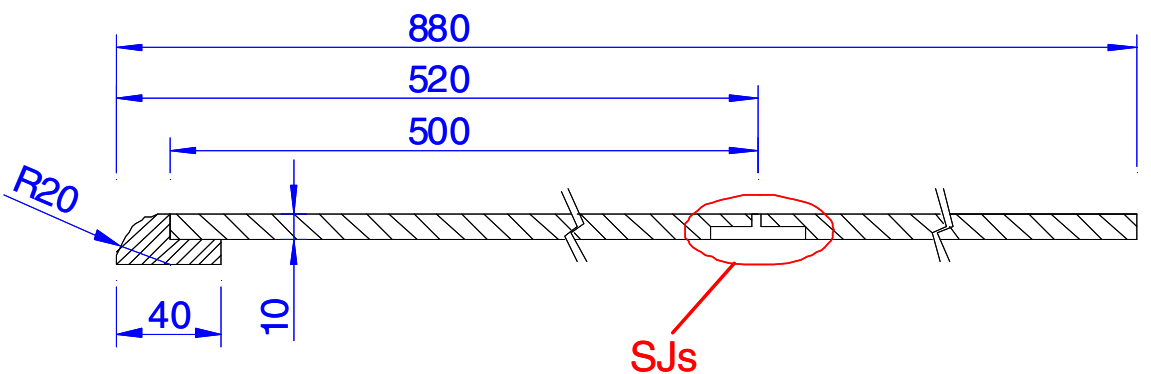

(b)

Fig. 4 Plexiglas wall with SJ array (a) general view, (b) schematic view.

channel at the Institute of Thermodynamics (IT) (described in Chapter 3.1.3).

\subsubsection{Circulation water channel}

Figure 5 shows the circulation water in which was put Plexiglas wall with SJ array. This vertical channel was made from Plexiglas. A pump pumps water to the top. Inside the channel, a Plexiglas plate with a SJ array is placed vertically.

\subsection{Experimental methods}

In this work, for experimental methods were used. Main part of the experiments was performed by means of particle image velocimetry. Auxiliary experiments were made with using of visualization, hot wire anemometry and laser Doppler vibrometry. 

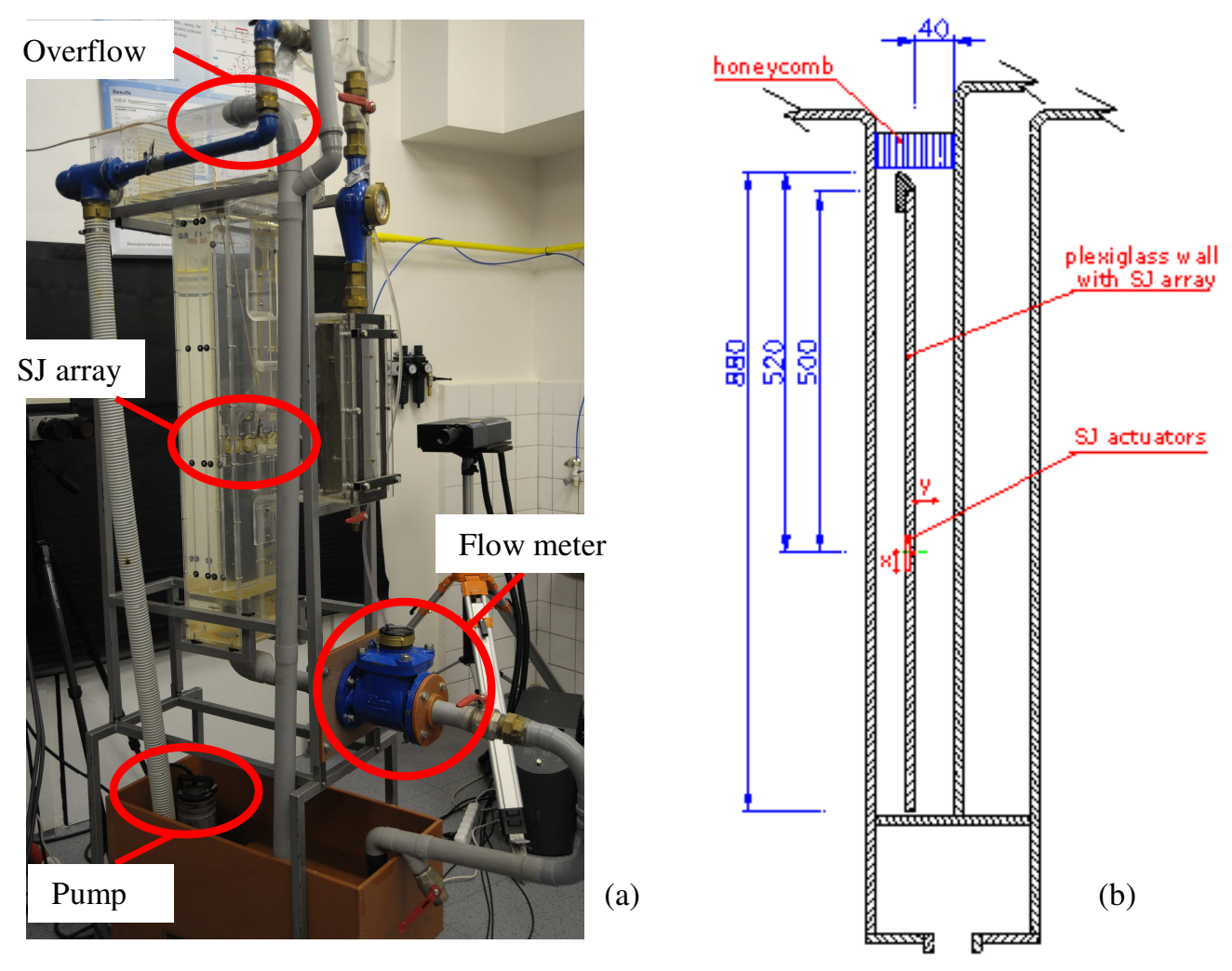

Fig. 5 Circulation water channel at IT CAS, v.v.i. (a) general view, (b) schematic view.

\subsubsection{Hot wire anemometry}

Hot wire anemometry (HWA) is one of the classical methods for measuring fluid velocities, including a fluctuating velocity component. It was used in this study to quantify SJ velocities in general and to evaluate the resonant frequency of SJ actuators in particular, [12].

The method is based on convective heat transfer from a heated body (hot wire or hot film probe) into a moving environment. The basic element of the measuring circuit is the probe, which is a wire fixed to the prongs of the carrier. The wire (typically tungsten) is heated by an electric current, [12].

For experiments, the DANTEC system was used: hotfilm probe 55P36, anemometer 90C10, bus bar NI_CA1000, and A/D converter NI-PCI-MIO-16E-1. The measured data was analyzed in StreamWare 3.01 and Microsoft Excel.

\subsubsection{Laser Doppler vibrometry}

Measurement of the oscillating PCT membrane is based on the Laser Doppler Vibrometry (LDV) principle. LDV is a non-contact vibration measurement technique utilizing the Doppler Effect. LDV permits the measurement of hot, miniature, or soft surfaces, even under water, without mass-loading. A laser Doppler vibrometer is based on a detection of the Doppler shift of coherent laser light that is scattered from a small area of a test object. The object scatters or reflects light from the laser beam, and the Doppler frequency shift is used to measure the component of velocity, which lies along the axis of the laser beam, [13].

The Ometron VH-1000-D (B\&K 8338) Portable Digital vibrometer is used to measure the velocity of the oscillating membrane. The main parameters of the vibrometer VH-1000-D are the following, [13]:

- Frequency range: $0.5 \mathrm{~Hz}-22 \mathrm{kHz}$.

- Measurement ranges (full scale (peak-peak)): $20 \mathrm{~mm} / \mathrm{s}$, $100 \mathrm{~mm} / \mathrm{s}, 500 \mathrm{~mm} / \mathrm{s}$.

- Spurious free dynamic range (SFDR): $>90 \mathrm{~dB}$.

- Best resolution: $0.02 \mu \mathrm{m} / \mathrm{s} / \mathrm{Hz} 0.5$.

The response of a PCT to a harmonic driving signal at frequency $f=15 \mathrm{~Hz}$ was measured. The LabView software was used to acquire and analyze the signal obtained from the Ometron vibrometer. Microsoft Excel was used for additional calculations and analysis.

\subsubsection{Tin ion visualization}

Tin ion visualization (or electrolytic precipitation method) is used for SJ array primary structures visualization. It helps to evaluate the basic character of the flow. This method is based on the tin ion transfer from an anode to a cathode in an electrolytic solution, described by Honji and Taneda [14]. For our experiments, a tin wire with a diameter $d=0.5 \mathrm{~mm}$ was used as the anode. A copper stick with a diameter $6 \mathrm{~mm}$ and length $140 \mathrm{~mm}$ was used as the cathode. A SJ nozzle was submerged into an electrolytic solution (mix of water and salt). The tin wire was attached close to the SJ orifice. Tin ions from the anode were released after DC voltage was applied. The particles were visible as a 
"white streak lines" with a very low sedimentation velocity. This method is very useful for velocities up to 5 $\mathrm{cm} / \mathrm{s}$, while the particle production is not sufficient at higher velocities, [15].

Experiments are performed at a frequency of $f=15 \mathrm{~Hz}$. For electrolysis, a voltage of $E=30 \mathrm{~V}$ and a current of $I=0.1$ A were used. For lighting, a laser for PIV system New Wave Geminy with output energy of $120 \mathrm{~mJ}$ in each pulse was used. By means of a cylinder optic, a thin light sheet was made, wherein the movement of tin ions was visible.

\subsubsection{Particle image velocimetry}

Particle Image Velocimetry (PIV) is used for channel flow and SJ array measurement. It is a non-intrusive laser optical measurement technique for research and diagnostics of flow, turbulence, microfluidics, spray atomization and combustion processes [16]. In PIV, the velocity vectors are derived from sub-sections of the target area of the particle-seeded flow by measuring the movement of particles between two light pulses.

The measurement fluid has to be transparent enough. The small particles are put into this flow environment. The particles are illuminated in the target area with a laser light sheet. A camera lens images the target area onto the sensor array of a digital camera. The camera is able to capture each light pulse in separate image frames. Once a sequence of two light pulses is recorded, the images are divided into small subsections called interrogation areas (IA). The interrogation areas from each image frame, $I_{1}$ and $I_{2}$, are cross-correlated. The correlation produces a signal peak, identifying the displacement. An accurate measurement of the displacement - and thus the velocity - is achieved with sub-pixel interpolation of the cross-correlation signal. A velocity vector map over the whole target area is obtained by repeating the cross-correlation for each interrogation area (with one vector for each interrogation area) over the two image frames captured by the camera [17].

For our experiments, a unit "Timer box" and DynamicStudio v.2.30 (DANTEC) software were used. All the control boards of the lasers, cameras, and timers of the input and output signals were placed directly into the main computer. DynamicStudio is a control and processing program for the unit Timer box. The system allows the computer to take on the role of a measuring center. Data communication between the PC, laser, and cameras runs through the internal protocols of the DynamicStudio control software and are impossible to modify. Starting signals are taken out by means of a National Instruments PCI-6601 counting measuring card into the unit Timer box with eight BNC connector outputs and two inputs (see figure 6).

Signals for the start of every flash lamp and Qswitching of the laser are led through one cable, whose position it is necessary to define - to Timer box to connect the starting trigger signal into two inputs.

Figure 7 shows the principle of basic synchronization. From the SJ signal generator, the TTL signal goes into
Timer Box

(80N76)

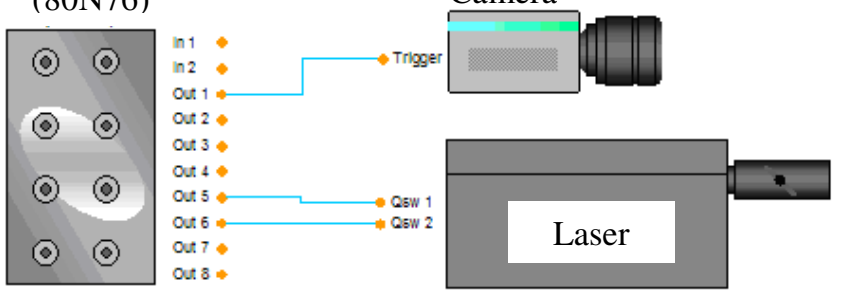

Fig. 6 A unit Timer box with signals for laser and camera.

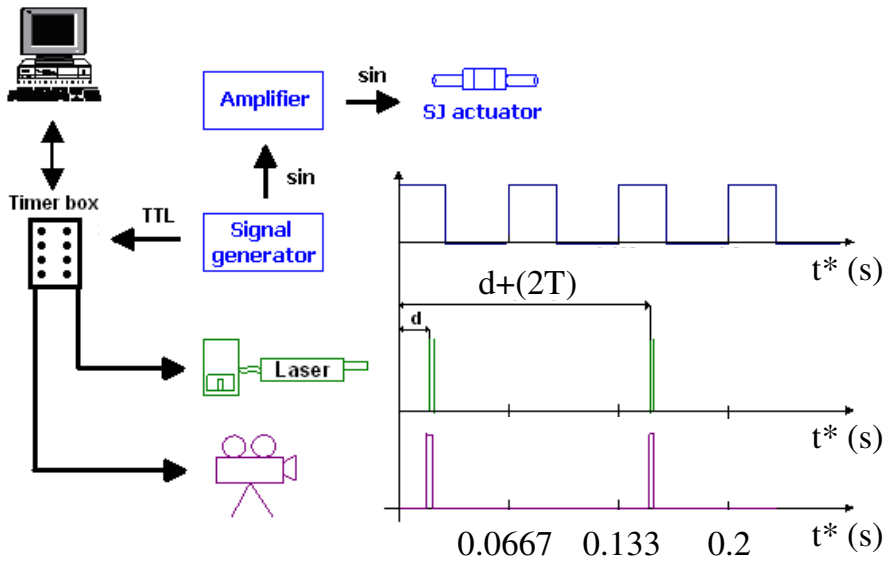

Fig. 7 Schematic of basic synchronization for PIV measurements ( $T$ is the period).

the PC (Timer box). The laser and camera start with the leading edge of the TTL. The starting point of measurement is changed during the SJ period and, therefore, the whole period is measured.

The delay inside the electronic synchronization devices does not exceed 3 s.

Two kinds of seeding particles were used: polyamide balls with diameters of $20 \mathrm{~m}$ (DANTEC) and fluorescent rhodamin coated particles with diameters of $10.2 \mathrm{~m}$ (Microparticles $\mathrm{GmbH}$ ). The particles were illuminated by a double pulse laser with a maximum $125 \mathrm{~mJ}$ per $10 \mathrm{~ns}$ pulse at a repetition rate of $2 \times 15 \mathrm{~Hz}$. The typical delay time between two pulses was $5 \mathrm{~ms}$.

The laser beam was expanded into a light sheet of approx. $1 \mathrm{~mm}$ thick. The image pairs were acquired using the HiSense MKI camera (DANTEC) with a spatial resolution of $1280 \times 1024$ pixels.

The resulting vector maps were averaged over 100 PIV records. Velocity vectors were determined by adaptive correlation using interrogation windows of $32 \mathrm{x}$ 32 pixels at a $25 \%$ overlap. Data processing was done using DynamicStudio 2.21 commercial software (DANTEC).

\section{Experimental results}

Next types of measurements were performed:

- Channel flow itself.

- SJ array in quiescent fluid.

- SJ array interacting with a channel flow. 
In this work, three definitions of time origin are used: $t^{*}$ is the time related to the leading edge of the TTL signal (used for LDV experiments and phase averaging of HWA results), $t^{* *}$ is related to the zero position of the membrane centre at the maximum extruded velocity, and $t$ is related to the beginning of fluid extrusion from the SJ actuator (i.e. at zero velocity of the fluid and maximum membrane displacement).

\subsection{Channel flow itself}

The first phase of the experiments was to visualize and measure the channel flow without the interaction of a SJ array. The reason for this experiment was to detect and set the laminar channel flow. For the first view of the flow, simple ink visualization was used - see figure 8 . The ink was inserted about $1 \mathrm{~m}$ upstream from the test section. The laminar parabolic profile of the channel flow corresponds quite well with the theoretical laminar parabolic profile.

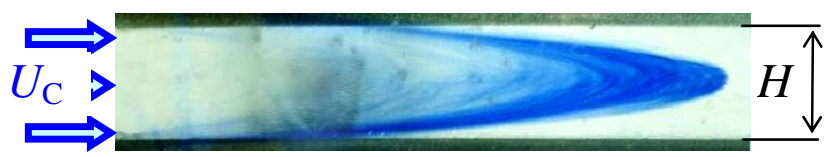

Fig. 8 Visualization of the laminar channel flow.

For a more precise measurement, the PIV method and polyamide particles were used. The typical time-mean velocity profile for the (main) channel flow without SJ interaction can be seen in Figure 9. It is easy to see that the measured profile compares very well with the parabolic Poiseuille profile (denoted by $U$ ):

$$
U=6 U_{\mathrm{C}}\left\lfloor y / H \quad(y / H)^{2}\right\rfloor,
$$

where the origin of the crosswise coordinate $y$ is chosen on the bottom wall of the channel - see figure 5(b).

The velocity profile was measured approximately in one-half of the channel length at location $x=0$ (see figure 5(b)). Note that the channel flow field at this point can be considered to be fully developed.

For evaluation of the Reynolds number, the timemean velocity through the channel cross section was considered as $U_{\mathrm{C}}=(2 / 3) U_{\max }$ (for the twodimensional case), and it is approximately $U_{\mathrm{C}}=0.0067 \mathrm{~m} / \mathrm{s}$. The Reynolds number of the channel flow was evaluated as $\operatorname{Re}_{\mathrm{C}}=536$.

To demonstrate the three-dimensionality of the real channel flow, Figure 10 shows the velocity profile $U(z)$. It is clear that the velocity profile is not fully developed (compared with figure 9). This confirms that the channel flow can be assumed to be $2 \mathrm{D}$, i.e. flow between two parallel walls.

\subsection{Frequency characteristic (using HWA)}

Finding a suitable SJ actuator nominal frequency was the first step in the experiments. The maximal time-mean velocity of the flow was achieved via the nominal frequency. For this reason, HWA was used.

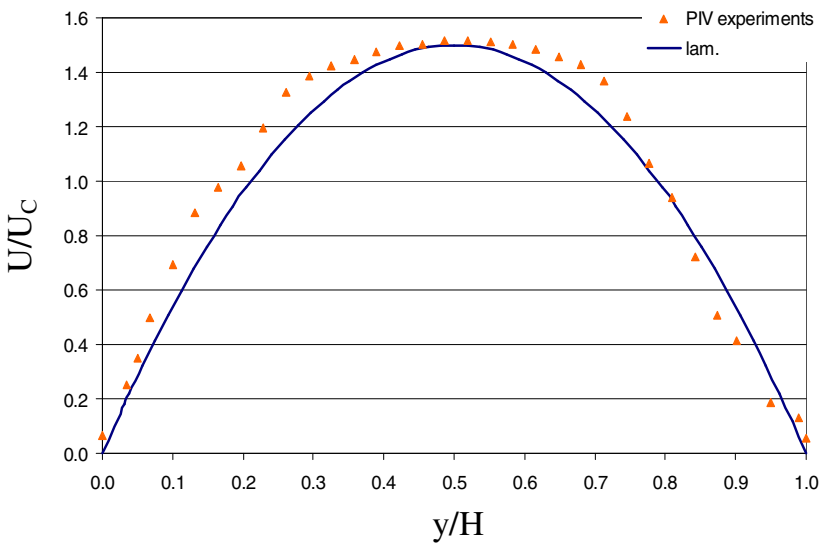

Fig. 9 Velocity profile of a channel flow without SJ.

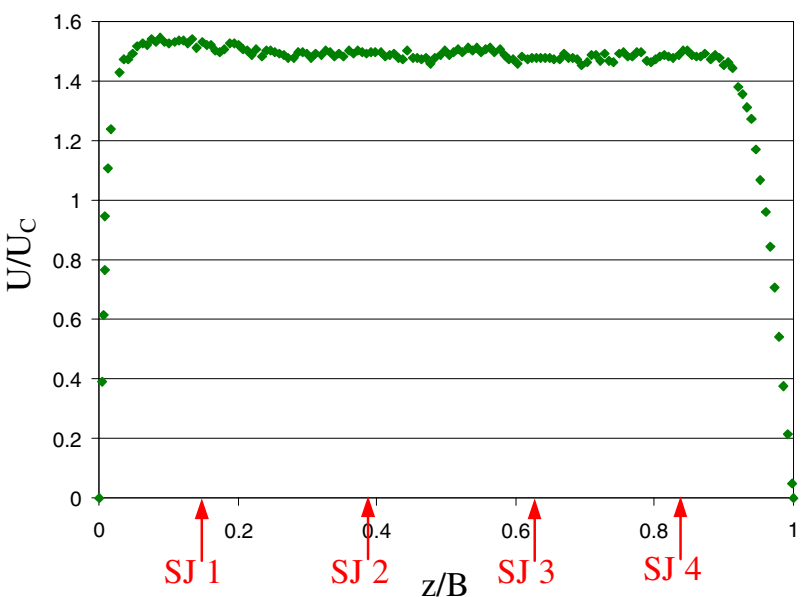

Fig. 10 Velocity profile of a channel flow without SJ.

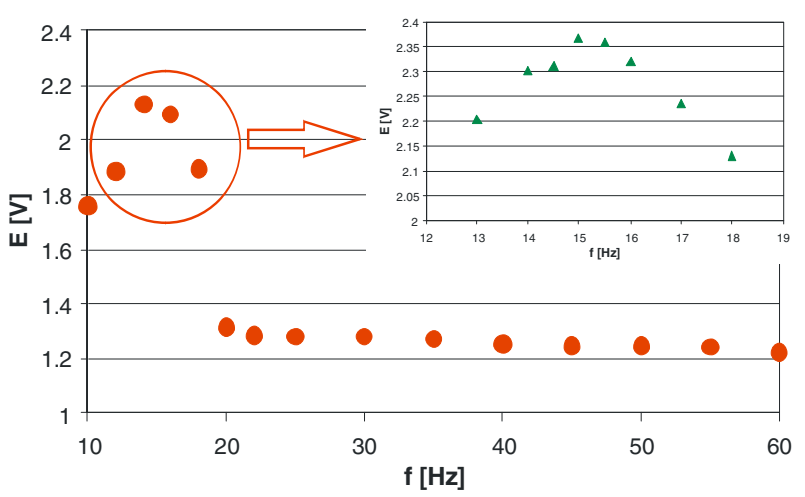

Fig. 11 Frequency characteristic of a SJ actuator.

Figure 11 shows the frequency characteristic in the form of the relationship between the HWA time-mean voltage and the frequency. The highest value of HWA voltage was achieved at $f=15 \mathrm{~Hz}$. Obviously, the discovered maximum frequency of $f=15 \mathrm{~Hz}$ was relevant to the first resonance of the actuator. Through theoretical calculation [18], $f=\frac{1}{2 C} \frac{D}{D_{\mathrm{m}}} \sqrt{\frac{k_{\mathrm{p}}}{L_{\mathrm{e}}}}$, the first resonance was estimated at $f=19.7 \mathrm{~Hz}$, which was in reasonable agreement with the experiment. The main reason for the difference was caused by 

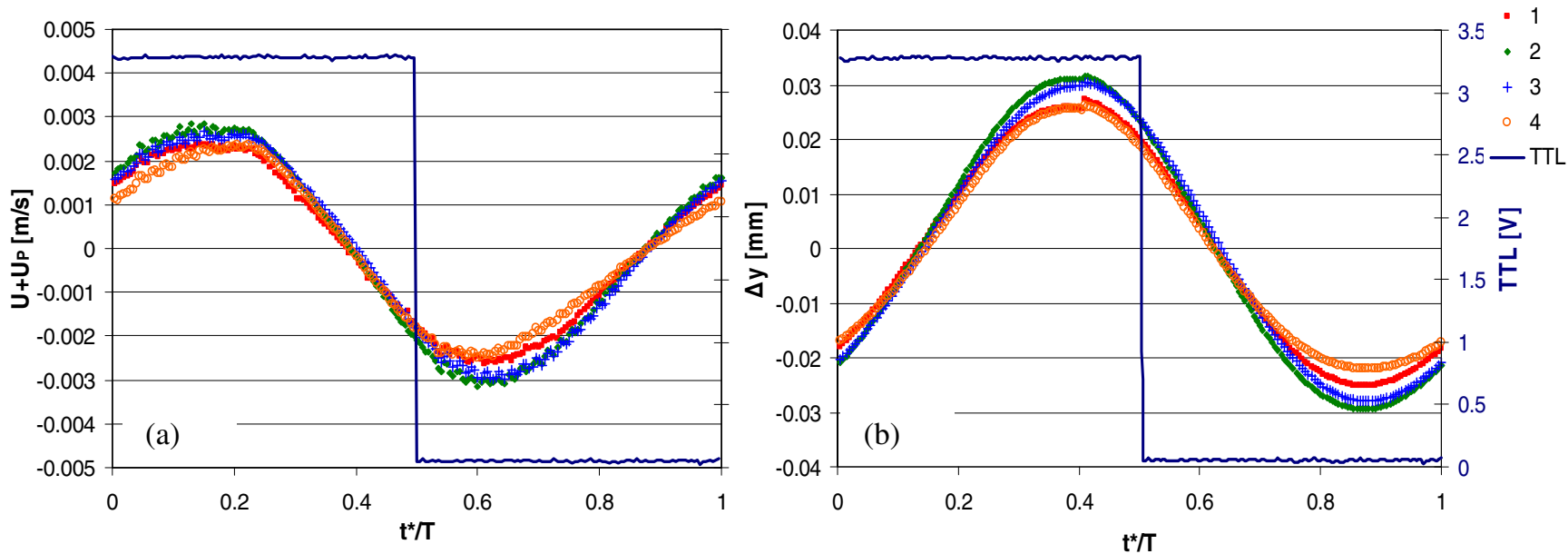

Fig. 12 Selected PCTs, similar power - oscillating membrane center in time (a) measured velocity, (b) evaluated displacement (results of LDV experiments).

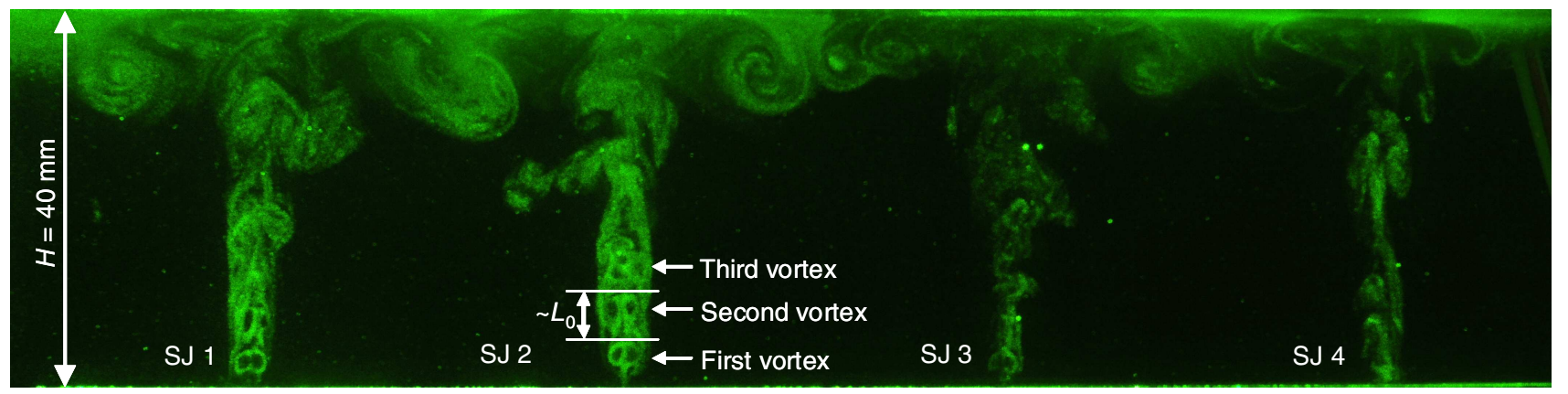

Fig. 13 Tin ion visualization, selected PCTs, similar power; $t / T=0.25$.
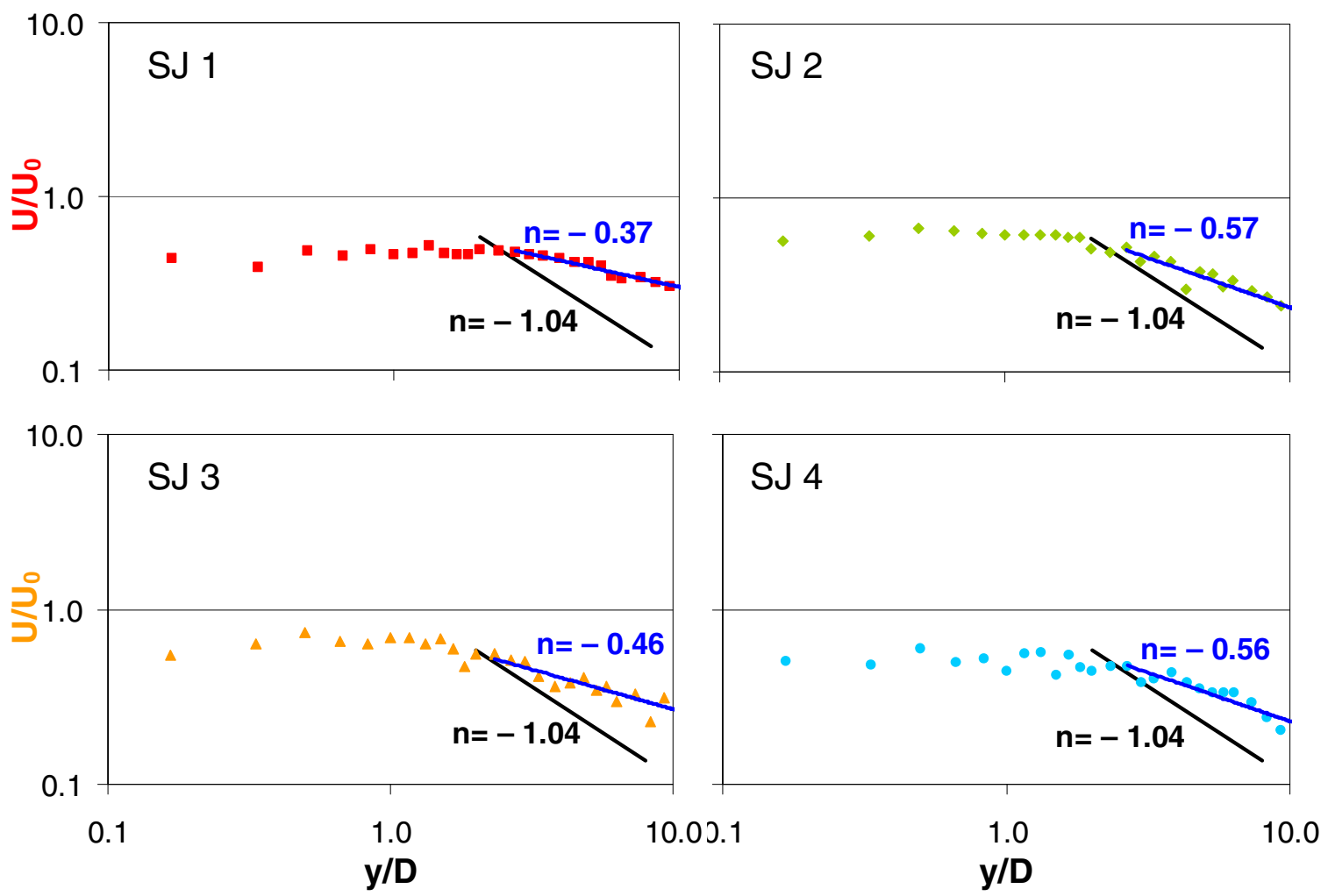

Fig. 14 Dependence of the time-mean velocity magnitude on the distance from the SJ actuator orifices on a logarithmic scale. 


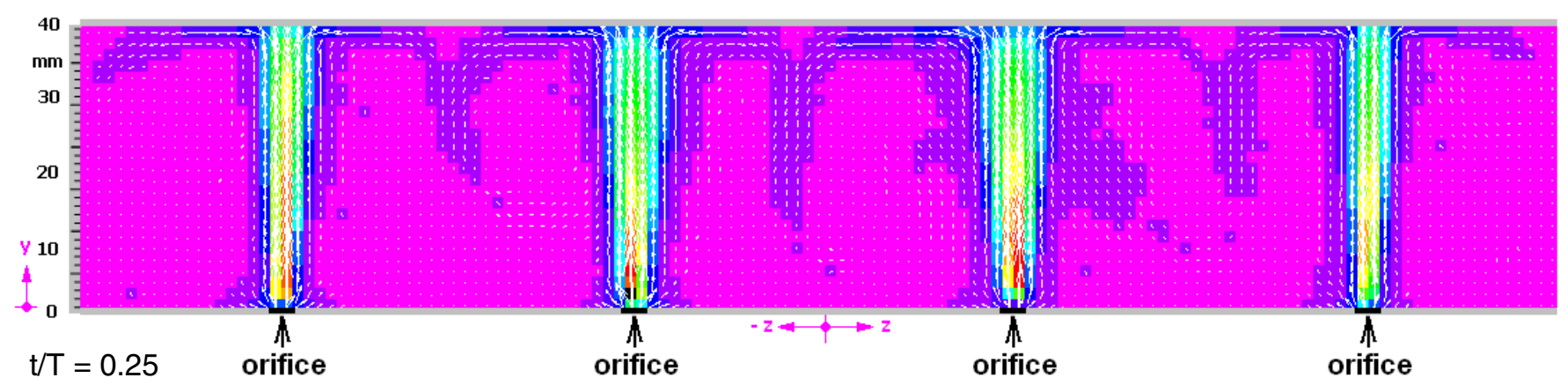

Fig. 15 SJ array in quiescent fluid - contours in discrete drawing style of the phase-averaged velocity magnitude (results of PIV experiments).

difficulties in evaluating PCT membrane stiffness, see [19].

In the end, the frequency $f=15 \mathrm{~Hz}$ was chosen as the nominal frequency of the SJ actuator.

\subsection{Synchronization of the piezoceramic system for SJ array (using LDV)}

The SJ array consisted of four SJ actuators located side by side across a channel. They were electrically connected in parallel. The actuating membranes were made out of piezoceramic transducers (PCTs). It was very important to use PCTs with identical or very similar characteristics. In spite of PCT producer guarantees of identical electrical properties, it is very important to take care during the phase shift of the particular PCTs used.

The set values for the experiments were the following: a true rms AC voltage of $19.5 \mathrm{~V}$, a true rms AC current of approx. $0.164 \mathrm{~A}$, and an apparent electric power of $0.8 \mathrm{~W}$ for each actuator $(3.2 \mathrm{~W}$ for the entire SJ array). The actuators working frequency was $15 \mathrm{~Hz}$.

Figure 12 shows measured velocity and evaluated displacement of PCT membrane centres. It demonstrates the same phase shift and a very similar velocity and displacement of the membrane centres.

From the membrane centre velocity, and considering continuity, the plug flow model of an extruded fluid column, the parabolic shape of the membrane deflection, the sinusoidal waveforms, the time-mean orifice velocity, and the Reynolds number of a particular SJ actuator were evaluated. The results were: $U_{0 \_1}=0.057 \mathrm{~m} / \mathrm{s}$, $U_{0 \_2}=0.066 \mathrm{~m} / \mathrm{s}, U_{0 \_3}=0.063 \mathrm{~m} / \mathrm{s}, U_{0 \_4}=0.053 \mathrm{~m} / \mathrm{s}$ and $\operatorname{Re}_{\mathrm{SJ}_{\_} 1}=170, \mathrm{Re}_{\mathrm{SJ} \_2}=199, \mathrm{Re}_{\mathrm{SJ} \_3}=190, \mathrm{Re}_{\mathrm{SJ} \_4}=158$.

\subsection{Flow visualization of a SJ array using the tin ion method}

For technical reasons, the visualization of a SJ array using the tin ion method was made in quiescent fluid. Figure 13 shows the influence of the individual jets and the vortex structures. The first three vortex rings behind the SJ actuator orifice are visible, as is indicated by arrows (demonstrated by SJ 2): The first one is near the actuator orifice, the second one drifts away from the orifice, and the third one is still clearly visible - obviously, the cascade process dissipates a bit further downstream.

Instantaneous pictures were recorded using a NIKON D300 camera, with an exposure time of $1.3 \mathrm{sec}$. For lightning, the laser for the PIV system New Wave Gemini, with output energy of $120 \mathrm{~mJ}$ per pulse, was used. The frequency of the SJ actuators and the laser system was set at $15 \mathrm{~Hz}$ and $3 \mathrm{~Hz}$, respectively. The tin wire was fixed $10 \mathrm{~mm}$ to the side of the orifices' axes. Electrolysis was running for only few seconds to make a tin ion "fog" but not long enough darken the water.

\subsection{SJ array velocity measurement using HWA}

Figure 14 shows the dependence of time-mean velocity on the orifice direction on a logarithmic scale, and it shows the slope of the line of each SJ. Note, that the bottom and top channel walls are located outside of the plotted area, namely at $\mathrm{y} / \mathrm{D}=0$ and 13.33 , respectively. It is a known fact that a non-influenced single $\mathrm{SJ}$ in a distant field has a line slope of $\mathrm{n}=-1.04$, which corresponds with the continual fluid jet (black line in a figure 14) - e.g. Schlichting and Gersten, [20]. In a SJ array, SJs are under the influence of mutual interactions, and the slope of the line has the exponent $n=\left(\begin{array}{ll}0.37 & 0.57\end{array}\right)$. A comparison with black line in a figure clearly demonstrates that the SJs in the array could not reach a developed stage with an exponent of $n \sim-1$.

\subsection{PIV experiments of a SJ array in quiescent fluid}

The delay time between the two laser pulses was $t=5 \mathrm{~ms}$ Due to the large size of the SJ array equipment, the images were scanned on two cameras. Only one camera did not record all four actuators in high-quality resolution. During post processing, these two pictures were composed into one and consequently processed. The Matlab program was used for composing.

Figure 15 demonstrates the results of the SJ array at $t / T=0.25$ (maximum extrusion from the cavity) in the form of phase-averaged velocity magnitude contours together with vectors, which show flow direction. The monitored plane was aligned to the collective centerline of all orifices. Figure 15 plotted at $t / T=0.25$ revealed the 


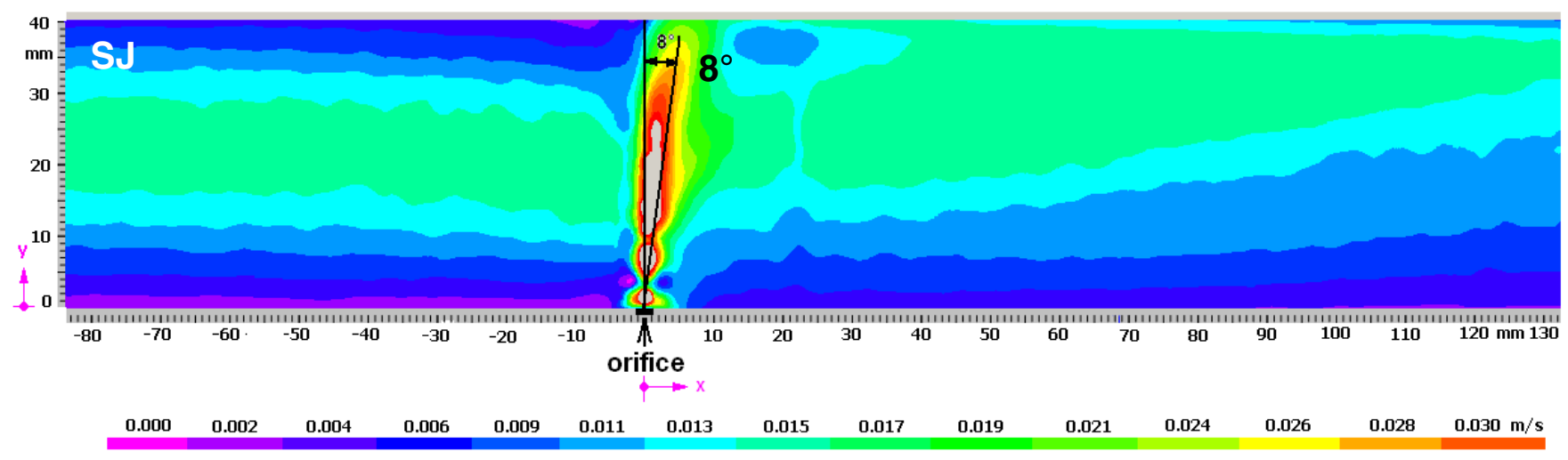

Fig. 16 Velocity magnitude contours of the channel flow interacting with a SJ array, measured on the plane of SJ 2 (i.e. $\mathrm{z}=-24 \mathrm{~mm}$ ); processing smoothing. Phase-averaged for $t / T=0.25$ (results of PIV experiments).

influence of individual SJs - a collision of the vortexes is seen in the upper third of figures.

\subsection{PIV experiments of a SJ array in a channel flow}

The delay time between the two laser pulses was $t=5 \mathrm{~ms}$. Experiments were performed in the plane of SJs 1, 2, 3, and 4 , i.e. $\mathrm{z}=(-72,-24,24$, and 72$) \mathrm{mm}$, with all SJs (1 4) operating. The results plotted in Figure 16 are in the form of velocity magnitude contours in time $t / T=0.25$ (maximum extrusion velocity) focused on SJ 2. There is visible small bending of a SJ and SJ reached the opposite wall $(y=40 \mathrm{~mm})$ at the location of approx. $x=8 \mathrm{~mm}$.

Figure 17 present phase-averaged velocity magnitude profiles at different distances from the orifices of the SJ array (all SJs were working) in the plane of SJs 2, i.e. $z=-24 \mathrm{~mm}$, respectively. These profiles are compared with theoretical laminar profile.

Figure 17(a) shows the velocity profile of the channel flow upstream of the SJ actuators. At a distance of $x=5 \mathrm{~mm}$, a small influence of the SJs was visible because $\left(U+U_{\mathrm{P}}\right) / U_{\mathrm{C}}$ reached a value of 0.4 at a distance of $y / H=0.01$.

Figure 17(b) show the influence of the velocity profiles on the top part of the channel. The velocity ratio $\left(U+U_{\mathrm{P}}\right) / U_{\mathrm{C}}$ even achieved a value of (2.7-3) at a distance of $x=10 \mathrm{~mm}$.

Further downstream, flow development was propagated downwards to the bottom channel wall, and the velocity profiles relaxed back towards parabolic profiles (Figures 17(c, d). Obviously, the parabolic profiles of the developed channel flow cannot be achieved in the rather short test section, limited by $x=350 \mathrm{~mm}-$ as is shown in Figures 17(e, $\mathrm{f}$, and $\mathrm{g}$ ).

The time-mean velocity through the channel and the synthetic jets' time-mean orifice velocities were evaluated at approximately $U_{\mathrm{C}}=0.0067 \mathrm{~m} / \mathrm{s}$ and $U_{0 \_1}=0.057 \mathrm{~m} / \mathrm{s}, \quad U_{0 \_2}=0.066 \mathrm{~m} / \mathrm{s}, \quad U_{0 \_3}=0.063 \mathrm{~m} / \mathrm{s}$, $U_{0 \_4}=0.053 \mathrm{~m} / \mathrm{s}$. The associated maximum Reynolds numbers were $\operatorname{Re}_{\mathrm{C}}=536$ and $\mathrm{Re}_{\mathrm{SJ}_{\perp} 1}=170, \mathrm{Re}_{\mathrm{SJ} \_2}=199$, $\operatorname{Re}_{\text {SJ } \_3}=190, R_{S_{S} \_4}=158$, respectively.

\subsection{Overview of the main results}

Table 1 compiles the main results of this work.

Table 1. Channel flow, and SJ array parameters for the presented experiments in water.

\begin{tabular}{|c|c|c|c|c|}
\hline \multicolumn{5}{|c|}{ Channel flow } \\
\hline $\begin{array}{l}U_{\mathrm{C}} \text { at } \mathrm{TU} / \mathrm{e} \\
(\mathrm{m} / \mathrm{s})\end{array}$ & 0.006 & \multicolumn{2}{|c|}{$\begin{array}{l}U_{\mathrm{C}} \text { at IT CAS } \\
(\mathrm{m} / \mathrm{s})\end{array}$} & 0.0067 \\
\hline $\operatorname{Re}_{\mathrm{C}}$ at $\mathrm{TU} / \mathrm{e}$ & 480 & \multicolumn{2}{|c|}{$\operatorname{Re}_{C}$ at IT CAS } & 536 \\
\hline \multicolumn{5}{|c|}{ SJ actuator nominal frequency } \\
\hline $\begin{array}{l}\text { Resonant } \\
\text { frequency } \\
\text { acquired from } \\
\text { the experiments } \\
(\mathrm{Hz})\end{array}$ & 15.0 & \multicolumn{2}{|c|}{$\begin{array}{l}\text { Calculated } \\
\text { resonant } \\
\text { frequency } \\
(\mathrm{Hz})\end{array}$} & 19.7 \\
\hline \multicolumn{5}{|c|}{ SJ array } \\
\hline & SJ 1 & \multicolumn{2}{|c|}{ SJ 2} & SJ 4 \\
\hline$U_{0}(\mathrm{~m} / \mathrm{s})$ & 0.057 & 0.066 & 0.063 & 0.053 \\
\hline$L_{0} / D=U_{0} /(f D)$ & 1.27 & 1.46 & 1.4 & 1.2 \\
\hline \multirow[t]{2}{*}{$\operatorname{Re}_{\mathrm{SJ}}=U_{0} D / v$} & 170 & 199 & 190 & 158 \\
\hline & SJ 1 & SJ 2 & SJ 3 & SJ 4 \\
\hline $\mathrm{St}=(1 / \pi) D / L_{0}$ & 0.25 & 0.22 & 0.23 & 0.27 \\
\hline $\begin{array}{l}\text { SJ deflection } \\
\text { flow } \varphi\left(^{\circ}\right) \text { on the } \\
\text { planes of SJ }\end{array}$ & 10 & 8 & 10 & 20 \\
\hline \multicolumn{3}{|c|}{$c_{\mathrm{U}}=\frac{1}{n} \sum_{i=1}^{n}\left(\frac{U_{0 \mathrm{i}}}{U_{\mathrm{C}}}\right)$} & \multicolumn{2}{|c|}{8.92} \\
\hline \multicolumn{2}{|c|}{$c_{\mathrm{Q}}=\frac{A}{A_{\mathrm{C}}} \sum_{n=1}^{n}\left(\frac{U_{0 \mathrm{i}}}{U_{\mathrm{C}}}\right)$} & & \multicolumn{2}{|c|}{0.03} \\
\hline$c_{\mathrm{M}}=\frac{5^{2}}{24}($ & $\sum_{i=1}^{n}($ & & \multicolumn{2}{|c|}{2.31} \\
\hline
\end{tabular}



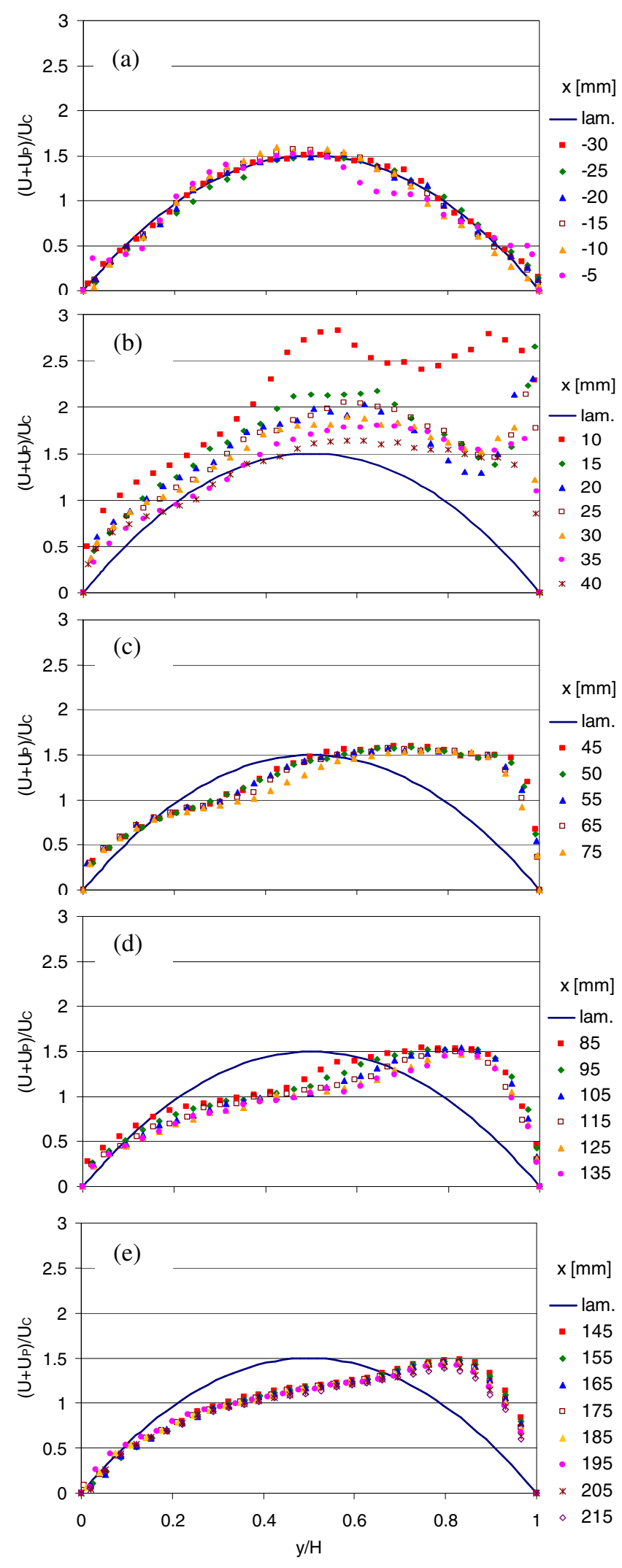

\section{Conclusions}

The investigation described in this work was focused on a laminar channel flow influenced by SJ array. This configuration can be effective in many micro-scale applications, e.g. in the cooling of microelectronics.
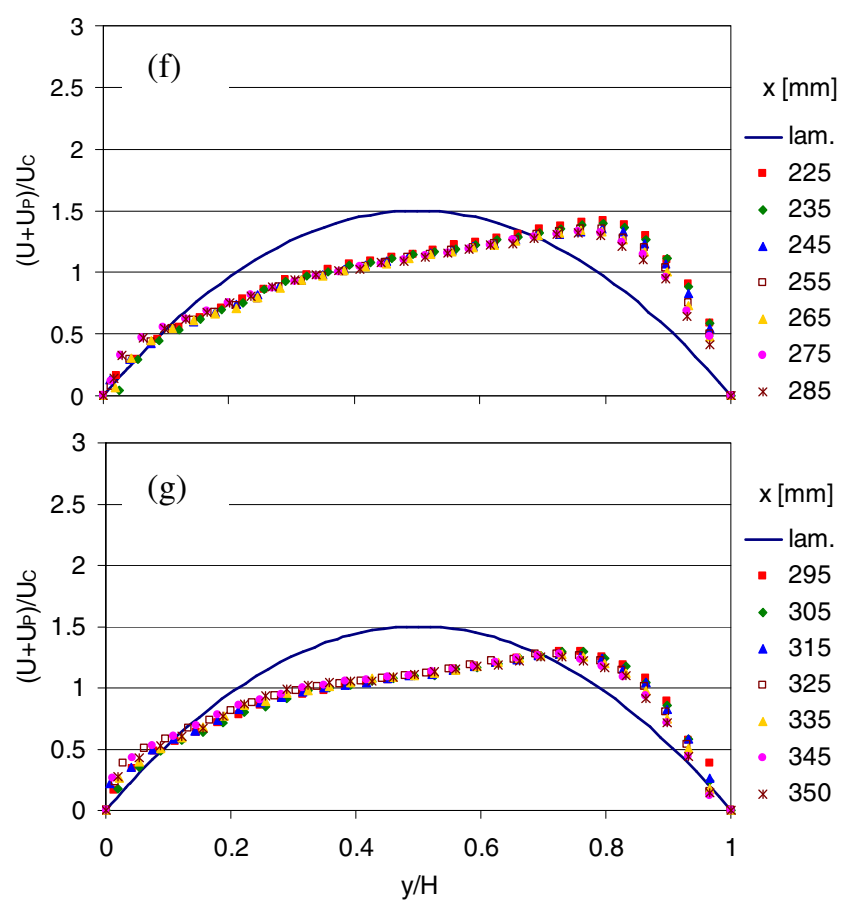

Fig. 17 Phase-averaged velocity magnitude profiles of the channel flow measured in the plane of SJ 2 (i.e. $z=-24 \mathrm{~mm}$ ), $t / T=0.25$ (results of PIV experiments).

All the experiments were performed using water as the working fluid.

Following experimental devices were used:

- Synthetic jet actuator, equipped with a sealed cavity and an actuating piezoelectric membrane (KINGSTATE KPS-100); actuator dimensions: orifice diameter $D=3.0 \mathrm{~mm}$, cavity diameter $D_{\mathrm{D}}=36 \mathrm{~mm}$.

- SJ array setup with dimensions: length, height, and width $L=210 \mathrm{~mm}, H=40 \mathrm{~mm}$, and $B=200 \mathrm{~mm}$, respectively. The upper wall was connected with four screws for easy removal.

- Plexiglas wall with SJ array, dimensions: length (length with start $) \times$ width $\mathrm{x}$ thickness $=(860(880) \times 200 \times 10)$ $\mathrm{mm}$.

- Circulation water channel at IT Prague with Plexiglas wall equipped with pump to raise water to the top.

For measurement of the problem, the following methods were used:

- Visualization with ink and tin ion to see the basic structure of the flow.

- Hot-wire anemometry in constant temperature mode to find the resonance frequency of a SJ actuator.

- Laser Doppler vibrometry was used for PCT behavior investigation and to find PCTs, which worked without phase shift, for the SJ array.

- Particle image velocimetry was used to investigate channel flow without the interaction of a SJ array, to investigate a SJ array in quiescent fluid, and to investigate a SJ array in a cross-flow. To properly complete the experiments, the SJ actuator (master) and the laser system (slave) had to be synchronized.

Following types of experiments were carried out:

- Channel flow without the interaction with the SJ array.

- SJ array in quiescent fluid. 
- SJ array interacting with the channel flow.

All the experiments with the SJ array were made at a constant true rms AC voltage of $19.5 \mathrm{~V}$, the true rms AC current was approx.0.164 A, and the apparent electric power was $0.8 \mathrm{~W}$ for each actuator $(3.2 \mathrm{~W}$ for the whole SJ array).

The working (resonant) frequency was $15 \mathrm{~Hz}$.

Based on the performed experiments, it can be stated that this configuration (on a micro-scale) is very useful for (micro) cooling applications.

Despite the experiments being carried out in three different laboratories, with different systems and setups, the results corresponded closely with each other and provided a perfect view of the SJ problem.

\section{Acknowledgments}

This work has been supported by grants GAASCR (IAA200760801) and GA CR (P101/11/J019).

\section{References}

1. R.K. Shah, A.L.London, Laminar flow forced convection in ducts (Academic press, Inc. New York, 1978)

2. B.L. Smith, A. Glezer, Phys. Fluids 10, 2281 (1998)

3. J.E. Cater, J. Soria, J. Fluid Mecha. 472, 167 (2002)

4. A. Glezer, M. Amitay, Annu. Rev. Fluid Mech. 34, 167 (2002)

5. B.L. Smith, A. Glezer, J. Fluid Mech. 458, 1 (2002)

6. D.A. Tamburelo, M. Amitay, J. of Turbulence 38 (8) (2007)

7. R. Mittal, P. Rampunggoon, Phys. Fluids 14, 1533 (2002)

8. V. Timchenko, J.A. Reizes, E. Leonardi, F. Stella, 13th International Heat Transfer Conference IHTC13, Sydney (2006)

9. H. Choi, 6th Symposium on Smart Control of Turbulence, Japan (2005)

10. M.B. Gillespie, W.Z. Black, C. Rinehart, A. Glezer, Trans. ASME, J. Heat Transfer 128, 990 (2006)

11. Z. Trávníček, V. Tesař, Int. J. Heat Mass Transfer 46, 3291 (2003)

12. H.H. Bruun, Hot wire anemometry (Oxford Univ. Press, 1995)

13. Portable vibrometer VH-1000-D (User manual, Ometron, 2005)

14. H. Honji, S. Taneda, M. Tatasuno, Rep. Res. Inst. Appl. Mech. 28, 83 (1980)

15. T. Vít: An investigation into selected types of flow. Habilitation thesis, Technical university of Liberec (2009)

16. V. Timchenko, J.A. Reizes, E. Leonardi, F. Stella, 13th International Heat Transfer Conference IHTC13, Sydney, NSW Australia, Aug. 13-18, 2006, Eds. G. de V. Davis and E. Leonardi, MIC-21

17. www.dantecdynamics.com

18. Z. Trávníček, A.I. Fedorchenko, A.B. Wang: Enhancement of synthetic jets by means of an integrated valve-less pump, Part I: Design of the actuator. Sensors and Actuators A 120 (1) (2005) 232-240.

19. P. Dančová, Ph.D. thesis (Technical University of Liberec, 2012)

20. H. Schlichting, K. Gersten, Boundary-Layer Theory (Springer-Verlag, Berlin, 2000) 